EPJ Web of Conferences 47, 03004 (2013)

DOI: $10.1051 /$ epjconf/20134703004

(C) Owned by the authors, published by EDP Sciences, 2013

\title{
YETI - search for young transiting planets
}

\author{
Ronny Errmann ${ }^{1, a}$, Ralph Neuhäuser ${ }^{1}$, Guillermo Torres ${ }^{2}$, Hiroshi Terada ${ }^{3}$, \\ Aglae Kellerer ${ }^{4}$, Gracjan Maciejewski ${ }^{5}$, Martin Seeliger $^{1}$ and YETI Team ${ }^{6}$ \\ ${ }^{1}$ Astrophysikalisches Institut und Universitäts-Sternwarte Jena, Schillergäßchen 2-3, \\ 07745 Jena, Germany \\ ${ }^{2}$ Harvard-Smithsonian Center for Astrophysics, 60 Garden St., Mail Stop 20, Cambridge \\ MA 02138, USA \\ ${ }^{3}$ Subaru Telescope, National Astronomical Observatory of Japan, 650, North A'ohoku Place, \\ Hilo, HI 96720, USA \\ ${ }^{4}$ Department of Physics, Durham University, South Road, Durham DH1 3LE, UK \\ ${ }^{5}$ Toruń Centre for Astronomy, Nicolaus Copernicus University, Gagarina 11, \\ 87-100 Toruń, Poland \\ ${ }^{6}$ All over the world
}

\begin{abstract}
We present our search for young transiting planets at ages of 2 to $20 \mathrm{Myr}$. Towards this goal, we monitor a number of young open clusters with the YETI network. YETI consists of 0.4-2 m-sized telescopes at different longitudes that observe continuously over timescales much longer than a night. In our first cluster Trumpler 37 we found more than 350 variable stars. Also two transiting candidates were found so far, for which follow-up is partly done. The first candidate turned out to be an eclipsing binary with an M-type companion. We describe the research done on these two transiting candidates.
\end{abstract}

\section{INTRODUCTION}

From the light curve of transiting planets it is possible to calculate planetary radius and inclination of the planetary orbit. Follow up with the radial velocity method then provides the planetary mass. Out of the nearly 300 transiting planets which are known so far only a few have young ages. The youngest star, which harbor transiting planets, is Corot 2 with age of 30-40 Myr from planetary radius respectively 130-500 Myr from rotation period and activity [1].

We monitor young clusters with ages of 2 to $20 \mathrm{Myr}$ to search transiting planets younger than any known to date. To reach near completeness, we observe with the YETI network (Young Exoplanet Transit Initiative, see Neuhäuser et al. [2]). YETI consists of small sized telescopes with mirror diameters of 0.4 to $2 \mathrm{~m}$, which are located at different longitudes all over the world so that nearly continuous observation are possible. Each cluster will be observed over 3 years, during 3 campaigns each year. The individual campaigns have lengths of 1 to 2 weeks.

\footnotetext{
ae-mail: ronny .errmann@uni-jena.de

* Based in part on data collected at Subaru Telescope, which is operated by the National Astronomical Observatory of Japan.

** Some of the data presented herein were obtained at the W.M. Keck Observatory, which is operated as a scientific partnership among the California Institute of Technology, the University of California and the National Aeronautics and Space Administration. The Observatory was made possible by the generous financial support of the W.M. Keck Foundation.

*** Partly based on observations made at the Centro Astronómico Hispano Alemán (CAHA), operated jointly by the Max-PlanckInstitut für Astronomie and the Instituto de Astrofísica de Andalucía (CSIC).

This is an Open Access article distributed under the terms of the Creative Commons Attribution License 2.0, which permits unrestricted use, distribution, and reproduction in any medium, provided the original work is properly cited.
} 


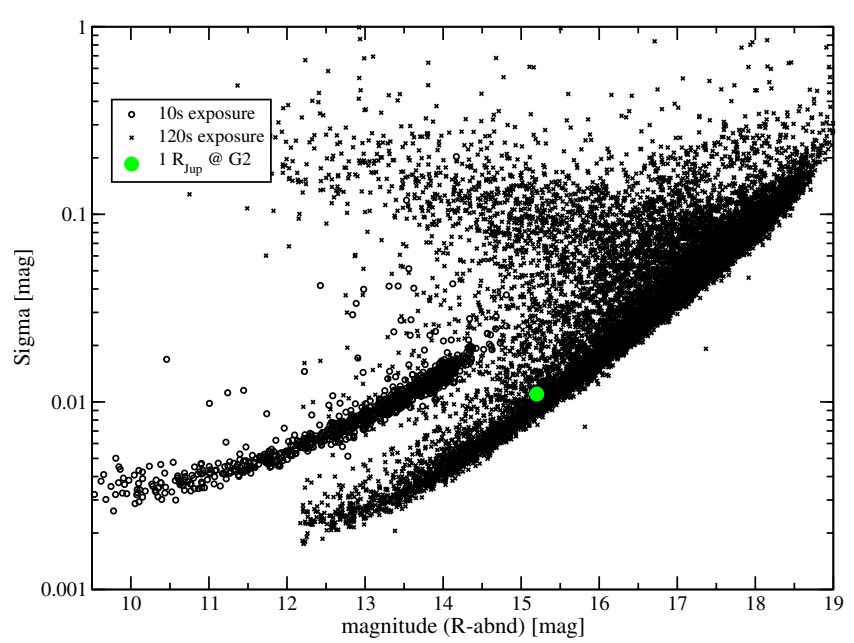

Figure 1. The photometric precision for the night August 302011 at the Jena $90 \mathrm{~cm}$ telescope. Observation were done with two alternating exposure times, resulting in two times 115 images. For each light curve the standard deviation sigma was calculated and plotted against the brightness of the star. Additionally, the signal of a Jupitersized planet around a solar-type star at distance of Trumpler 37 is included.

Trumpler 37 was the first cluster monitored with our network. The $\sim 4 \mathrm{Myr}$ old cluster [3], [4] at a distance of $\sim 870 \mathrm{pc}$ [5] contains at least 774 stars with high membership probability [6]. Trumpler 37 has an apparent diameter of $1.5^{\circ}$, which is not covered by all YETI telescopes. Because of its distance and position in the sky, a lot of foreground and background stars contaminate the cluster.

\section{DATA REDUCTION AND ANALYSIS}

The data from the individual telescopes were reduced in a standard way: bias, dark, and flat-field correction was applied. For 24,500 different stars in the area of Trumpler 37, we extracted light curves using aperture photometry with an optimized aperture for each night and telescope. Differential photometry was done afterwards, using an artificial standard star, which was built from the most constant light curves [7].

Figure 1 shows the photometric precision of one night for the Jena $90 \mathrm{~cm}$ telescope. We reach enough precision to be able to detect Jupiter-sized objects transiting solar type stars. Young planets are expect to be bigger in size, so if we assume a cut-off of $30 \mathrm{mmag}$, we could detect those on more then 5500 stars in Trumpler 37 [2].

We found more than 350 variable stars in Trumpler 37. One such variable star is shown in Fig. 2. We also investigate in these stars, another variable star (GM Ceph) is presented in [8]. Among the variable stars are two transiting candidates.

\section{TRANSITING CANDIDATES AND FOLLOW UP}

The phase folded light curves of the transiting candidates are shown in Fig. 3. The second star was slightly active with period of $\sim 9$ days and an overall brightness change of $\sim 15 \mathrm{mmag}$, this was removed for the folded light curve. The parameters for both stars are given in Table 1.

For the first star no membership investigation is available, and for the second, a low proper motion membership had been determined [9]. The positions in the color-magnitude diagram indicate high membership probability, so further investigations were done by us. For the second candidate they are still in progress, so we focus in the following section on the first star. 


\section{Hot Planets and Cool Stars}

Table 1. Parameters of the transiting candidates. Given are the significant decimals.

\begin{tabular}{lll}
\hline Candidate & star 1 & star 2 \\
\hline Brightness of primary (R-band) & $15.1 \mathrm{mag}$ & $13.4 \mathrm{mag}$ \\
Spectral type & $\mathrm{G} 2$ & $\mathrm{G} 4$ \\
Period & $1.36491 \mathrm{~d}$ & $0.736867 \mathrm{~d}$ \\
Transit depth (R-band) & $54 \mathrm{mmag}$ & $10.6 \mathrm{mmag}$ \\
Inclination & $86.5^{\circ}$ & $71.2^{\circ}$ \\
Companion to primary radius & 0.1965 & 0.1084 \\
\hline
\end{tabular}

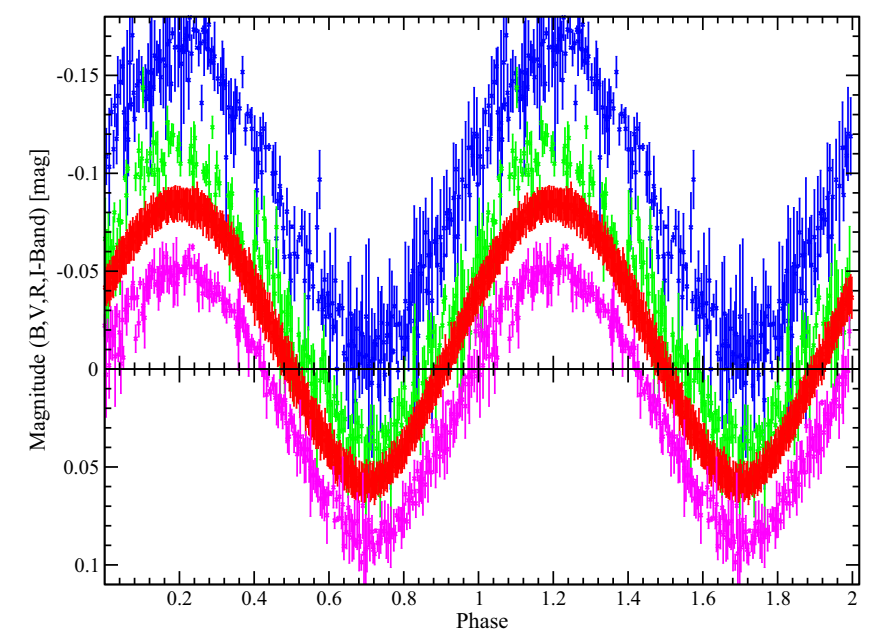

Figure 2. The star $2 M 21405752+5722093$ showing rotation with period of $0.224945(3) \mathrm{d}$. The individual curves show from top to bottom the $B, V, R$, and $I$ band data. Only Jena data were used. The number of data points in $B, V$, and $I$ are ten times less than in $R$ band, causing the bigger errors of the former bands. Amplitude variations between the different bands are visible. The star's proper motion makes it a highly proper motion member of Trumpler 37 [9].
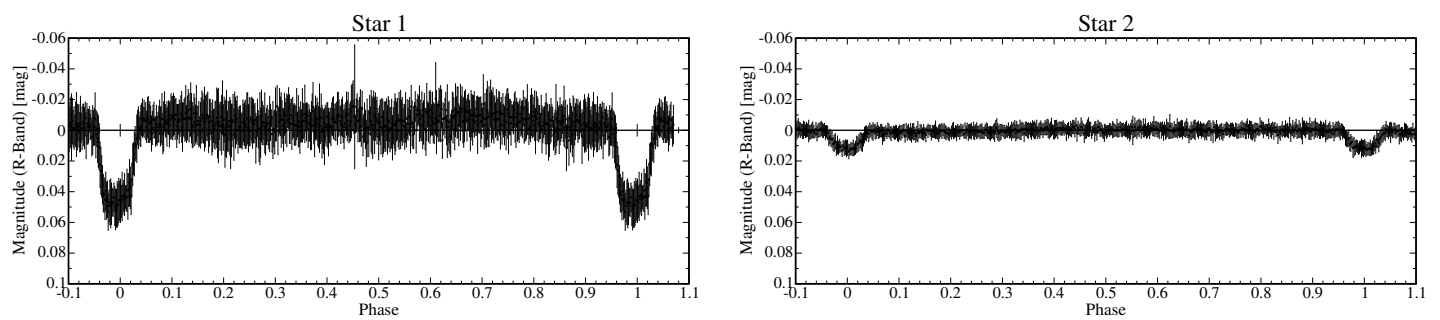

Figure 3. The phase folded and binned light curves for our two transit candidates in Trumpler 37. More than 10,000 data points of the Jena $90 \mathrm{~cm}$ telescope were used for the light curves of each star.

We checked for blended stars, which were unresolved in our telescopes, using the IRCS instrument [10] and the adaptive optics system AO-188 at the Subaru telescope. Images were taken in $J, H$ and $K$-band. Dark and flat-field correction was done. Afterwards, sky subtraction and dithering was applied. The final, color image is shown in Fig. 4. There are a few faint sources next to the main star, but all of them are too faint to produce a $50 \mathrm{mmag}$ deep signal, even if they are full eclipsing binaries.

Radial velocity variations were determined from HIRES spectra [11], obtained at one of the Keck telescopes. The extracted and calibrated spectra were cross-correlated against artificial spectra. Figure 4 

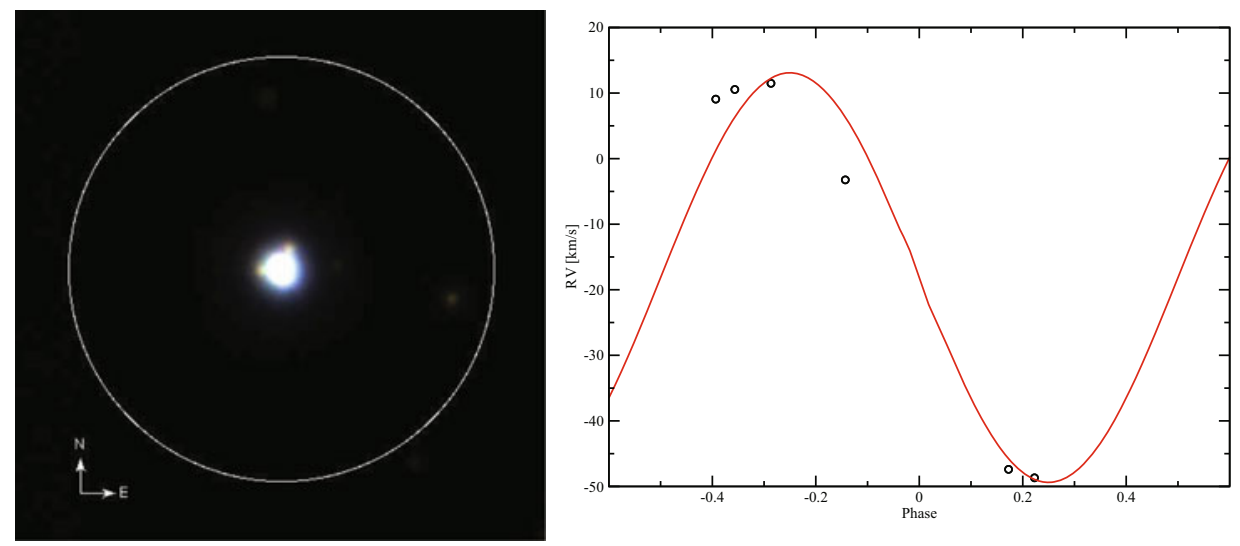

Figure 4. Left: Subaru image of the area around star 1. The 3.5" big circle corresponds to the smallest point-spread functions of the YETI telescopes. Right shows the radial velocity data of star 1 from Keck and the best fit, assuming a circular orbit. The periodicity and ephemeris were obtained from the light curves.

shows the phase folded measurements, using the periodicity of the light curve. Overplotted is the best circular fit with an amplitude of $\sim 31 \mathrm{~km} / \mathrm{s}$, resulting in a mass of an M-type star in spectral type. The systematic deviations of the observations suggest a possibly eccentric orbit. The system's center-of-mass velocity is consistent with membership in Trumpler 37 [12].

For both candidates low resolution spectra were taken with CAFOS at the $2.2 \mathrm{~m}$ telescope on Calar Alto. Unfortunately, both show small Lithium absorption, making the youth and therefore membership in the young cluster uncertain.

\section{OUTLOOK}

We are still working on improving the data reduction. We are also working on an improved transit detection algorithm, using a Bayesian approach to identify variations in intensity. The clusters 25 Ori, IC 348, Collinder 69 and IC 2602 are also monitored by us. We expect to find many more transiting candidates.

We thank Andrew W. Howard for the reduction of the Keck spectra. RN and RE would like to thank DFG for support in the project NE 515 / 33-1/2 and 34-1. RE also thanks the Abbe-School of Photonics for support.

\section{References}

[1] Guillot, T., Havel, M., A\&A 527, A20 (2011)

[2] Neuhäuser, R., Errmann, R., Berndt, et al., AN 332, 547 (2011)

[3] Kun, M., Kiss, Z. T., Balog, Z., Handbook of Star Forming Regions, Vol. 1: The Northern Sky (ASP Monograph Series, San Francisco, 2008) 136

[4] Sicilia-Aguilar, A., Hartmann, L. W., Hernández, J., Briceño, C., Calvet, N., AJ 130, 188 (2005)

[5] Contreras, M. E., Sicilia-Aguilar, A., Muzerolle, J., Calvet, N., Berlind, P., Hartmann, L., AJ 124, 1585 (2002)

[6] Errmann et al., AN, submitted 


\section{Hot Planets and Cool Stars}

[7] Broeg, C., Fernandez, M., Neuhäuser, R., AN 326, 134 (2005)

[8] Chen, W. P., Hu, S. C.-L., Errmann, R., et al., ApJ 751, 118 (2012)

[9] Marschall, L. A., van Altena, W. F., AJ 94, 71 (1987)

[10] Kobayashi, N., Tokunaga, A. T., Terada, H., et al., Porc. SPIE 4008, 1056 (2000)

[11] Vogt, S. S., Allen, S. L., Bigelow, B. C., et al., Porc. SPIE 2198, 362 (1994)

[12] Sicilia-Aguilar, A., Hartmann, L. W., Fürész, G., Henning, T., Dullemond, C., Brandner, W., AJ 132, 2135 (2006) 